\title{
Innovation and growth through local and global interaction
}

\author{
Rainer Andergassen ${ }^{1,2,4}$, Franco Nardini ${ }^{3}$, Massimo Ricottilli $^{1,2 *}$ \\ ${ }^{1}$ Department of Economics, University of Bologna \\ P.zza Scaravilli 2, 40126 Bologna (Italy) \\ ${ }^{2} \mathrm{CIG}$, Centro Interdipartimentale Luigi Galvani
}

${ }^{3}$ Department of Mathematics for the Economic and Social Sciences, University of Bologna Viale Filopanti 5, 40126 Bologna (Italy)

${ }^{4}$ RCEA, Rimini Centre for Economic Analysis (Italy)

June 2008 .

Abstract. This paper investigates the research and development activity of heterogeneous and rationally bounded firms. The latter conduct this activity through in-house research and by collecting information originating in other firms' spillovers. Thus, research and development activity owes both to independent searching and to interaction diffusing information. We study the conditions under which this idiosyncratic effort yields effects that have either local, system-wise negligible impacts or cumulate to generate significant aggregate ones. In the latter case, global effects feed back upon the incentive to innovate and therefore on the strength of local interaction as well as on autonomous research efforts. It is these dynamic forces that we model. We compare cases in which significant aggregate effects do emerge with cases in which they do not and study their outcome on innovation-directed investment and on long-term growth.

JEL classification: O31;O41;

Keywords: Economic growth; Innovation and diffusion; Innovation waves;

\footnotetext{
*E-mail addresses: rainer.andergassen@unibo.it, franco.nardini@unibo.it, massimo.ricottilli@unibo.it.
} 


\section{Introduction}

It is a well established fact that technical progress is an engine of growth. Economic history and theory concur to indicate that the reason lies with profit opportunities pried open by innovationgenerated productivity growth and the spurt in economic activity that thereby follows ${ }^{1}$. They also point out that innovations are themselves the result of a complex effort involving one sided investment in autonomous research as well as interaction between firms as they attempt to capture informative spillovers that they, in turn, willingly or unwillingly, broadcast. It is, indeed, this two-pronged, complex activity that renders technical progress largely endogenous to firms' endeavours. Although an innovation may involve any part of a firm's activity or process, economic historians (David, 1990; Rosenberg, 1976; Mokyr, 1992) have highlighted the crucial role played by machine-producing industries in raising productivity and fostering a radical transformation of production systems. This occurrence much owes to the fact that embodying technical advances in these firms' output is a powerful, if not the main, tool of competition and finally market dominance. Thus, capital goods producing firms appear as the fundamental protagonists of the innovation process. New devices, machines, tools, whole new manufacturing systems, in fact the introduction of entirely novel technological paradigms are in great part due to the innovative efforts of firms which then supply those lying forward in the production chain: the final goods producers. Their technological and, ultimately, economic success depends on the breadth and depth of their learning and searching process, a process that normally requires costly investment in human capabilities. It is important to note that firms so engaged are necessarily specialised and thus each one attempting to innovate is cognitively bound within a fairly well defined and possibly narrow knowledge domain. They are not, however, islands barred from all communication. On the contrary, they benefit from the structural externalities afforded by the system as a whole; namely, the educational system providing general competence and skills, institutions able of crafting an

\footnotetext{
1 See, for example, Aghion, Howitt (1992), (1998) and Romer (1990).
} 
innovation-friendly environment, a thriving scientific context able of fostering the development of viable technologies and, crucially, the all-important entrepreneurship without which innovative activity is left wonting. None of these favourable structural elements can be exploited without resourceful striving. In very broad terms, innovation requires information that can be contrived both by an independent search and by interacting with other firms. Both are crucial features of the race to innovate. While the former consists in a self generated attempt such as it occurs in research and development departments, the latter is an effort at gleaning useful information from other firms. This information collection activity is clearly made possible by the fact that spillovers are available although through a costly search that largely depends on information interaction. As literature on technological convergence has shown (Dosi, 1988; Fai and von Tunzelmann, 2001; Rosenberg, 1976) this kind of information on cutting-edge technologies comes from firms that belong to other industries and that have been able to innovate in their own sector of activity ${ }^{2}$. They are, in this sense, leaders that to some extent broadcast technologically novel features to cognitively nearby industries. What may, in fact, appear as a wholly new technology in a given industry may feature principles pioneered elsewhere in the economy, its information leading to implementation in different industries through spillovers. Yet, this externality can be captured only by means of industrious and clearly costly observation requiring effort and resources. Recent literature has stressed the role of firms' interaction in spreading technological paradigms (Andergassen, Nardini, Ricottilli, 2005, 2006). In this context, two relevant aspects of firms' innovative activity must be stressed. The first concerns the limits they encounter as a consequence of their specialisation, the latter being a factor bounding the cognitive distance that can effectively be covered when searching for information. It is quite clear that the specificity of their acquired skills and, more generally, of their cumulated knowledge sets a hurdle to the type and amount of information they can actually glean by scanning their technological environment. In this sense, firms are better assumed as rationality bounded agents availing themselves of limited capabilities

\footnotetext{
2 See also Helpman Trajtenberg (1998) and Jaffe and Trajtenberg (2002).
} 
as to the amount of information they can process and the number of sources they can observe. These matter-of-fact conjectures suggest that learning and searching carried out by firms, their interaction and networking are activities that take place in a neighbourhood the cardinality of which is rather small in terms of their overall number. Thus, while an economy can be viewed as a complex network of cognitive relations, the latter are better construed within neighbourhoods of limited size. The second aspect concerns what may be termed the contiguity principle. Whilst information is easily broadcast and easily captured within neighbourhoods of fairly homogeneous knowledge, it can nevertheless travel abroad since links between them are likely to exist. Literature on networks has repeatedly highlighted this point (Watts and Strogatz, 1998; Albert and Barabasi, 2002; Jackson, 2008). What matters in this respect is the density of knowledge-wise contiguous industries and thus of neighbourhoods that they make up. Economies endowed with a dense grid of industries perform better in spreading information than economies whose industries are cognitively sparse and far apart. This paper attempts to provide a model in which these aspects are taken in due consideration by positing that information interaction be a process that leads to a time constrained, gradual collection of elements of information contributed by different sources. It also intends to show that the joint impact of self generation of new, technology-relevant, knowledge and localised firms' interaction leads to a critical process that eventually produces an avalanche of innovations, that is to say to a number of firms that become empowered by sufficient information to devise a new capital good that embodies a productivity-augmenting technological novelty. As mentioned, this occurrence is not the consequence of a purely random event and is not scot-free but, although randomness is part of the process, it clearly requires substantial investment in, mainly, human resources. Thus, capital-goods producing firms intending to innovate have to sustain relevant expenditure in the hope of cashing in future rewards. It is, indeed, the expectation of future profits that drives investment: profits that accrue thanks to the higher productivity that innovations afford and to the monopolist strength the would-be innovator will eventually enjoy.

Search-for-innovation results may have either local or aggregate outcomes: we specifically 
distinguish these two cases. On the one hand, a productivity rise, a sector-specific occurrence, if interaction is weak may have system-wise negligible effects. On the other hand, if interaction is strong, it becomes a momentous event that determines far-flung effects at a global level. Observers and policy-makers hold as a rather well documented fact that productivity increases are shared out as to keep income shares roughly constant, a fact that bears relevant consequences on the equilibrium prices of those industries that are involved in the innovation wave. If the process of information gathering and searching is one of local interaction, it may nevertheless have an important impact on the system and determine a global interaction effect that then feeds back locally. To show how this happens, the paper models the impact of sectorial changes on the economy as a whole. It is important to note in this respect that expected profits depend on the likelihood of an innovation and on the probability that the consequently gained market power be maintained. In a dynamic context, in which the innovation race goes on, this is possible provided that the next waves of innovation occur in sectors other than in the would-be monopolist's own. Yet, profits depend on prices whose magnitude is fixed by the innovation-induced productivity rise and, in turn, determine aggregate expenditure in final goods. For any given labour force, while expected profits set current investment consisting of highly specialised employment, the grey matter behind innovations, and then its size in the sector engaged in producing intermediate goods, aggregate demand defines it in final good sectors establishing a complex feed-back that weaves together global and local interaction.

The plan of the paper is as follows. The second section takes into account all these important features by modelling the economy as being made up by industries each of which has a final good sector that does not engage in any innovation process but that fully absorbs the new technologies embodied in capital goods that, for simplicity's sake, are modelled as purely intermediate ones. The latter are produced by a backward lying sector that, for every practical purpose, appears as fully and vertically integrated. The first is dealt with as being competitive, populated by an accordingly very large number of firms, whilst the second is seen as featuring a monopolist actively 
engaged in manufacturing but challenged by a potential innovator that, if successful, will drive him or her out of the market. The third section tackles the fundamental question of innovation dynamics highlighting the role of firms' interaction and the search-for-innovation process at a microeconomic level and then we explore their macroeconomic implications. Firstly, the size of innovation-oriented employment is generated as a solution of a maximization problem of the probability of getting the required information either through independent, in-house search or through interacting with other firms, or both. Secondly, aggregate diffusion dynamics are derived by modelling firms' interaction resorting to a mean-field approach and by characterising the propagation mechanism. Section four provides a solution for the size of investment in the human resources required to innovate as a consequence of profit expectations and thus of the innovation rate. The model is solved for the steady state equilibrium: we both characterize the aggregate information diffusion process and distinguish between cases in which interaction is not sufficiently strong to generate significant innovation waves at the system level and cases in which significant aggregate outcomes appear. ${ }^{3}$ In this context, a solution for the equilibrium allocation of specialised manpower to be employed to generate an innovation is then provided. It is the solution of this problem that highlights the coupling of local and global interaction. It is shown that no appreciable effects emerge when interaction is weak whilst a trade off exists between global, profit enhancing effects and the local, profit dampening effect due to innovations displacing current monopolist leaders when it is strong. Section five investigates the implication of search and information diffusion on the long-term growth rate and price behaviour. As customary, conclusions bring the paper to an end. All proofs are in the Appendix.

\footnotetext{
3 The emergence of aggregate fluctuations resulting from microeconomic localised interaction is known in literature as self-organised criticality (see, for example, Bak, Tang, Wiesenfeld, 1988; Vespignani and Zapperi, 1998 and Bak, 1996). Economic applications include inventory dynamics and business cycles (Bak, Chen, Scheinkman, Woodford, 1993) and technology diffusion and adoption (Arenas, Díaz-Guilera, Pérez, Vega-Redondo, 2002).
} 


\section{The Economy}

The economy we wish to discuss is portrayed as a simple system of many industries each producing a single final product. Let these industries be designated by $j=1,2 \ldots . J, J$ being large. Each such industry is, in turn, made up by two distinct sectors: the first by a multitude of firms that compete with each other in actually producing the final good while the second produces and supplies them with a vital intermediate input (which embodies the frontier technology) and furthermore carries out research and development. Although each such sector may in principle accommodate several firms, we assume that the latter features just two. One is an undisputed technological leader that owns the frontier technology, enjoys the returns of a successful but past innovation and therefore has a monopolistic position supplying final producers whilst the other is a follower that carries out a significant technological searching activity, collects information through costly observation and stands a positive probability of becoming the next successful innovator and thus of driving out the present day incumbent.

\subsection{The final good sector}

All perfectly competing firms avail themselves of the latest technology consisting of an intermediate good, purchased from the currently in-charge monopolist, as well as of unskilled labour. Let $y_{j, t}^{s}$ stand for industry $j$ final good output at time $t$. Inputs enter output according to constant coefficients and such that

$$
y_{j, t}^{s}=\min \left\{a_{j, t} x_{j, t}, b_{j, t} l_{j, t}\right\}
$$

where $\frac{1}{a_{j, t}}$ is the amount of intermediate good required per unit of output. Likewise, $\frac{1}{b_{j, t}}$ is the quantity of unskilled labour per unit of output; $x_{j, t}$ is the intermediate total quantity and $l_{j, t}$ that of labour. Subscripts are as above defined. Let $n_{j, t}$ be the total number of innovations in sector $j$ introduced until time $t$, each of them having raised productivity by $\lambda \in(0,1)$. Production coefficients accordingly result as

$$
a_{j, t}=a_{j, 0} e^{\lambda n_{j, t}} \text { and } b_{j, t}=b_{j, 0} e^{\lambda n_{j, t}}
$$


which are then normalised such that, to provide symmetry in the economy's initial conditions, $a_{j, 0}=a_{0} ; b_{j, 0}=b_{0}$. We denote by $T(j, k)$ the time in which the $k$-th innovation has been introduced in sector $j$; it is straightforward that $0<T(j, 1)<\ldots<T\left(j, n_{j, t}\right) \leqslant t$.

Efficient production decisions require that $y_{j, t}^{s}=a_{j, t} x_{j, t}=b_{j, t} l_{j, t}$ and thus

$$
l_{j, t}=\frac{a_{0}}{b_{0}} x_{j, t}
$$

Profits accruing to final good producing firms at time $t$ are notionally defined as:

$$
\pi_{j, t}=p_{j, t} y_{j . t}^{s}-w_{t} l_{j, t}-u_{j, t} x_{j, t}
$$

where $p_{j, t}, u_{j, t}, w_{t}$ are the final good price, that of the intermediate good and the unskilled labour wage rate respectively. The assumption that perfect competition prevails in the final good sector implies that final goods prices adjust as to render profits equal to zero: $\pi_{j, t}=0$. It also implies that firms therein engaged be compelled to adopt the latest frontier technique lest they be pushed into loss making and driven out of the market.

\subsection{The intermediate goods sector}

The firm in industry $j$ 's intermediate good producing sector is, as long as its technological leadership lasts, a monopolist that employs a production function using only specialised labour according to a constant productivity coefficient $\eta$. We call this specialised, engineers' and skilled workers', employment with $h_{j, t}^{x}$ and, thanks to its specialised nature, labour engaged in this sector is paid a wage rate $w_{t}^{h}$ which is normally higher than the unskilled rate $w_{t}$.

Assumption 1. The wage rate of specialised labour, $w_{t}^{h}$, is a multiple $\omega>1$ of unskilled manpower's $w_{t}: \frac{w_{t}^{h}}{w_{t}}=\omega$.

Supply of the intermediate good is then defined by:

$$
x_{j, t}=\eta h_{j, t}^{x}
$$


This intermediate output carries the most up-to-date technology, allows the producer to enjoy a monopoly and earns a positive profit when sold to the final good sector :

$$
\pi_{j, t}^{x}=\left(u_{j, t}-\frac{w_{t}^{h}}{\eta}\right) x_{j, t}
$$

\subsection{Prices, profits and expenditure}

Given their market power, firms operating in the intermediate good sectors set their price $u_{j, t}$ as to keep it as a constant proportion of production costs that in this case are merely wages paid to specialised labour. We accordingly make the following assumption:

Assumption 2. Intermediate goods producers set their price according to a constant mark up $c$ :

$$
u_{j, t}=(1+c) \frac{w_{t}^{h}}{\eta}
$$

The mark up, $c$, is the magnitude upon which successful innovators rely upon to recoup costs incurred in the search-for-innovations process and generate profits. Since profits in the final goods sector are driven to zero, $u_{j, t}$ also follows straightforwardly from (3) as:

$$
u_{j, t}=p_{j, t} a_{j, t}-w_{t} \frac{a_{0}}{b_{0}}
$$

and is positive provided that revenue per unit be higher than labour cost per unit, a condition

that is met if $\frac{p_{j, t} a_{j, t}}{w_{t}}>\frac{a_{0}}{b_{0}}$. Taking into account (6) and (7), the final good price $p_{j, t}$ is immediately obtained in terms of the amount of labour that is directly and indirectly embodied into one unit of final output given the respective wage rates and weighted by the mark up $1+c$ :

$$
p_{j, t}=\frac{1}{b_{j, t}} w_{t}+\frac{1}{\eta a_{j, t}} w_{t}^{h}(1+c)
$$

In turn, from (5) profits are:

$$
\pi_{j . t}^{x}=c \frac{w_{t}^{h}}{\eta} x_{j, t}
$$


This economy's aggregate income is spent on final goods that, as indicated above, are competitively produced. In equilibrium, supply and demand must match. A very simple assumption is made to insure that this happens: each one of the $J$ industries composing this economy receives a fixed fraction of total aggregate demand ${ }^{4}$. Given the scope of this paper, for simplicity's sake this fraction is set such that each industry gets a share $\frac{1}{J}$ th of aggregate income $Y_{t}$. Thus, equilibrium requires

$$
y_{j, t}^{s}=y_{j, t}^{d} \equiv \frac{1}{J} \frac{Y_{t}}{p_{j, t}}
$$

and $\sum_{j}^{J} y_{j, t}^{s} p_{j, t}=Y_{t}$. Once each industry's final output is determined, the intermediate good input requirement is derived:

$$
x_{j, t}=\frac{1}{a_{j, t}} \frac{1}{J} \frac{Y_{t}}{p_{j, t}}
$$

As it can be seen, the time pattern of aggregate final output has a straightforward impact upon each industry's intermediate demand and thus on the latter revenue and, ultimately, profits.

Taking into account (9), (11) and Assumption 1, the above yields

$$
\pi_{j, t}^{x}=\frac{c}{\frac{\eta}{\omega} \frac{a_{0}}{b_{0}}+(1+c)} \frac{1}{J} Y_{t}
$$

This equation indicates that the intermediate producer's profits are a function of total final output and that their share is constant in time. Profits are earned at time $t$ : it is important to note that it is on their expectation that the current monopolist had, in the past, invested in a search for innovation; furthermore, it is on the expectation of future profits that the current follower is equally undertaking investment in the hope of a new innovation that will eventually oust the leader from its dominant position. They depend, in each industry $j$, on aggregate final output $Y_{t}$ which, in turn, depends on the supply and demand of all other industries. Accordingly, innovations occurring in any of them, by increasing productivity, have an impact on the aggregate and consequently feed

4 This assumption can be rationalised by considering a representative consumer with a logarithmic utility function

$$
U\left(y_{1, t}^{d}, \ldots, y_{J, t}^{d}\right)=\sum_{j=1}^{J} \ln \left(y_{j, t}^{d}\right)
$$

where $y_{j, t}^{d}$ indicates consumption (demand) of product $j, j=1, \ldots, J$. 
back on all industries, causing cross sectional spillovers. The implication is that global interaction (through aggregate output) has an impact on current profits, on the expectation driving current investment and thus on local interaction among firms and industries that will eventually bring about a new innovation.

\subsection{The labour supply}

The way we have designed this economy, its activity is made up of final aggregate outputs requiring unskilled labour as well as of sectors in which successful innovators, enjoying monopoly power, attend to the production of intermediate goods and in which followers search for the next innovation, both employing highly skilled manpower. We consider that the available and fully employed skilled and unskilled labour supply, designated by $\mathcal{L}$, is constant over time. Employment is then split threefold between a specialised labour force engaged either in the search and development of innovations or in the manufacturing of intermediate, innovation-embodying, goods and unskilled manpower hired to produce the final ones. Let $H_{t}^{I}$ and $H_{t}^{x}$ indicate the former and $L_{t}$ the latter, $H_{t}^{I}=\sum_{j=1}^{J} h_{j, t}^{I}, H_{t}^{x}=\sum_{j=1}^{J} h_{j, t}^{x}$ and $L_{t}=\sum_{j=1}^{J} l_{j, t}$ being their distribution in the various industries. Clearly, $\mathcal{L}=H_{t}^{I}+H_{t}^{x}+L_{t}$. It is a well understood fact that unskilled labour cannot be easily turned into a highly specialised labour force. This paper, however, attempts to deal with processes that take place in the long run and thus we assume an engaging learning and training process; indeed, a costly one given Assumption 1. As later shown, the partition of the available labour force will depend on the innovation investment effort and on the effective demand that is in consequence consistently determined in the industries' final good sectors.

Since each industry $j$ final sector employs labour in proportion to its supply which is a share $\frac{1}{J}$ of aggregate income, its employment amounts to:

$$
l_{j, t}=\frac{1}{J} \frac{1}{b_{j, t} p_{j, t}} Y_{t}
$$

and summing through the $J$ industries, taking into account (8) and Assumption 2, aggregate 
output can be set to equal

$$
Y_{t}=w_{t}^{h}\left[\frac{1}{\omega}+\frac{1}{\eta} \frac{b_{0}}{a_{0}}(1+c)\right] L_{t}
$$

On account of the symmetry assumptions we have made, we observe that by using (8) and (13) we can express industry $j$ employment as a fraction $\frac{1}{J}$ of the total $L_{t}$

$$
l_{j, t} \equiv l_{t}=\frac{1}{J} L_{t}
$$

Turning now to the intermediate sector, its employment is related to the final sector's as:

$$
h_{j, t}^{x} \equiv h_{t}^{x}=\delta l_{t}
$$

where $\delta=\frac{b_{0}}{\eta a_{0}}$, and by summing through the $J$ industries

$$
H_{t}^{x}=\sum_{j=1}^{J} h_{j, t}^{x}=\delta L_{t}
$$

a constant multiple of the total unskilled labour supply.

The magnitude of expected profits at any point of this period of time can be surmised by resorting to (12) and (14) yielding

$$
\pi_{t}^{x}=\pi_{j, t}^{x}=\frac{1}{\eta} \delta l_{t} w_{t}^{h} c=h_{t}^{x} w_{t}^{h} c
$$

\section{Information search and diffusion}

In this economy, leadership of any specific industry and the implied monopolistic position can be challenged through innovation. As discussed above, at any point in time an intermediate producer, by owning rights to a technology introduced in the past, enjoys monopoly powers at the same time as another firm, a follower, is engaged in innovation-aimed investment to oust the leader if and when it will be devised. Innovation requires a process of searching and learning. Quite generally, the framework in which the latter takes place is a knowledge space made up by the frontier technologies that have appeared thanks to past innovations in all the industries belonging to this economy. Seen in this perspective, this space is made up of specific know-how, skills and 
capabilities contributed by industries that appear as repositories of idiosyncratic knowledge. It is important to note, however, that diversity and specificity resulting from consolidated division of labour are not necessarily a barrier to the spreading of relevant information provided that cognitive proximity exists. This feature of an economic system, namely inter-industry contiguity, is assured by a high number $J$ of industries such that each be surrounded by a cognitively reachable neighbourhood capable of passing on useful information. It is, in fact, important to consider that knowledge specificity and its related technological capabilities are instrumental in setting each firm in a given neighbourhood. While information spreads with an ease that we purport to measure by an index of interaction strength, as explained below, within neighbourhoods that are on average cognitively homogeneous, their proximity determines overlapping in neighbourhood membership thus providing channels of information transmission. It follows that a large number of industries relatively to the economy's size insures a contiguity that generates knowledge contagion and a rich, albeit indirect, exchange of information. On the contrary, where industries are sparse and stand far apart in terms of their specific cognitive characteristics and neighbourhoods are effectively isolated clusters, information remains locally bound and spreads but little in the system as a whole. The assumption of a large $J$ insures that the leading firms that happen to have been successful in bringing about an innovation become an important source of information for followers in cognitively nearby industries: they broadcast technological principles that can be exploited by those that are actively searching.

\subsection{The search process}

Followers, by assumption one in each industry, carry out a search for information which leads to a successful innovation once the information gathering process is completed. Our assumption is that for a successful innovation $S$ informational bits have to be collected ${ }^{5}$. The information gathering process combines two overlapping activities. The first consists of an in-house endeavour

\footnotetext{
${ }^{5}$ Andergassen et al. (2006) investigate the case of innovations with heterogeneous informational content.
} 
to contrive innovative events by carrying out autonomous research. Large and sophisticated firms are indeed able to support full fledged research and development departments but this activity needn't necessarily be so formalised: it is nevertheless important to note that employment of highly specialised labour is required to this end.

Let $h_{j, t}^{I_{1}}$ denote the specialised workforce engaged in in-house $\mathrm{R} \& \mathrm{D}$ activity in sector $j$, we then assume that, at the single firm level, the independent discovery of information is a Poisson distributed process with arrival rate $\mu f_{j}\left(h_{j, t}^{I_{1}}\right)$, where $f_{j}\left(h_{j, t}^{I_{1}}\right)$ is the contribution of the $j-t h$ firm. $\mu$ is a scale factor. As shown further on, this scale factor allows to separate the exogenous arrival of new information from the endogenous one. The purpose of this separation is to highlight the dynamics of technological progress as they occur as a consequence of firms' interaction. For simplicity's sake, we set $f_{j}()=.f_{i}()=.f(),. \forall i, j$ and hence we define $q_{j, t}=q\left(h_{j, t}^{I_{1}}\right)=\mu f\left(h_{j, t}^{I_{1}}\right)$. Over a small time period $d t$, for the economy as a whole the average probability that such an information come to pass by active searching supported by specialised manpower is assumed to be $\widehat{q}_{t} d t$, where $\widehat{q}_{t}=\widehat{q}\left(h_{1, t}^{I_{1}}, \ldots, h_{J, t}^{I_{1}}\right)=\mu \frac{1}{J} \sum_{j=1}^{J} f\left(h_{j, t}^{I_{1}}\right)$.

The second activity owes to information gathering, that is to the catching of spillovers provided by active monopolists who have achieved to innovate. As argued above, this process is enacted within a cognitive neighbourhood in which information has a concrete possibility to spread. In order to glean informational bits, innovations introduced by neighbouring sectors have to be processed by employing highly competent and clearly costly personnel. To streamline the formal exposition we assume that the cardinality of the cognitive neighbourhood coincides with the number of bits to be collected to reach up to successful completion $S$. Note that this assumption implies no loss of generality. The propagation of information across sectors depends on the number of potential neighbours capable of broadcasting useful information as well as on its dissipation, that is on information accumulated by a single firm but which cannot be passed on to other firms. Hence we normalise the number of firms capable of receiving useful information spillovers to be equal to the number of informational bits firms have to accumulate, and, as we will show in greater 
detail later, consider the strength of interaction as a control parameter for the diffusion process.

As long as gathered information is still incomplete, it cannot be passed on to other firms: only perfected innovations are actually observable. Diligent observation, however, is not necessarily crowned with success; rather, it depends on the strength of interaction between firms, a measure of their capabilities to understand each other, to explore technological novelties in industries that are neighbours defined in terms of their cognitive proximity and that engage in different productive activities. Let $\epsilon_{i, j, t}=\epsilon_{i j}\left(J, h_{i, t}^{I_{2}}\right) \geq 0$ designate the measure of the strength of interaction between industry $i$ and $j$, that is the average quantity of informational bits which firm $i$ is able to retrieve from its neighbour $j$ when the latter introduces a new innovation. As mentioned above, the density of industries in the system is a crucial parameter in defining it but it also depends on the amount of resources that the would-be innovator is prepared to invest for this specific task. These resources are mainly specialised personnel, $h_{i, t}^{I_{2}}$, the cost of which is measured by a wage rate that is higher than the one paid to unskilled manpower. Yet, in very large economies, i.e. for very large $J$, the strength is likely to dissipate in the process of interaction. Observation of real economies indicate, however, that there are structural elements providing cohesion and bestowing them the capability to interact, a property which we dub the 'industrial district effect'. To simplify exposition and tractability, we find it is expedient to assume interindustry symmetry $\epsilon_{i j}()=.\epsilon($.$) ,$ $\forall i, j$. Thus, we assume that the strength of interaction of each firm with each neighbouring one is the same.

Let $\widehat{\epsilon}_{t}=\widehat{\epsilon}\left(J, h_{1, t}^{I_{2}}, \ldots, h_{J, t}^{I_{2}}\right)=\frac{1}{J} \sum_{j=1}^{J} \epsilon\left(J, h_{j, t}^{I_{2}}\right)$ denote the average aggregate spill-over strength. Thus, given $h_{j, t}^{I}$ each sector has to allocate $h_{j, t}^{I_{1}}$ and $h_{j, t}^{I_{2}}$ in order to maximize the probability of receiving an informational bit, with $h_{j, t}^{I_{1}}+h_{j, t}^{I_{2}}=h_{j, t}^{I}$.

Assumption 3. (i) Let the industry specific probability of achieving an exogenous innovative event over a small time period $d t$ be $q_{j, t} d t$ where $q_{j, t}=q\left(h_{j, t}^{I_{1}}\right), q^{\prime}>0, q^{\prime \prime}<0, q(0)=0$ and $\lim _{h \rightarrow 0} q^{\prime}(h) \rightarrow \infty ;\left(\right.$ ii) let the industry specific spillover strength be $\epsilon_{j, t}=\epsilon\left(J, h_{j, t}^{I_{2}}\right)$, where $\epsilon_{J}>0$, 
$\epsilon_{h^{I_{2}}}>0, \epsilon_{h^{I_{2}}, h^{I_{2}}}<0, \lim _{h^{I_{2} \rightarrow 0}} \epsilon_{h^{I_{2}}}\left(J, h^{I_{2}}\right)=\infty, \lim _{J \rightarrow \infty} J \epsilon_{h^{I_{2}}}\left(J, h^{I_{2}}\right)>0$ and $\epsilon(J, 0)=0$.

Note that the denser is an economic system hallmarked by different industries, the higher is the strength of interaction just as it is higher, the higher is employment hired to carry out the informative search. The former, however, is assumed to weaken as the latter becomes larger: a realistic assumption of decreasing information returns to specifically hired manpower.

\subsection{Aggregate innovation spillovers}

Given the above described search process, we model aggregate information diffusion by resorting to a mean-field approximation (see Vespignani and Zapperi, 1998). Having posited $S$ as the number of informational bits required for a successful innovation, let $\rho_{k}$ be the density of firms having accumulated $k$ bits at time $t$, for $k=0,1, \ldots, S$. Thus, $\rho_{S}$ is the density of firms innovating at time $t$ : each of them propagates information to its $S$ cognitive neighbours and leaves the scene to a would-be innovator that begins the information-gathering process from state 0 . The probability that a firm be in state $S-1$, the critical one preceding an innovation, is given by $\rho_{S-1}$. Thus, provided that a firm in some sector is able to innovate and broadcast vital information, yet another innovation is introduced, subject to the strength of interaction $\epsilon$, with probability $\epsilon \rho_{S-1}$. Since the neighbourhood cardinality is $S, \epsilon S \rho_{S-1}$ firms, on average, succeed in introducing a new innovation. This event leads to further propagation of new information and possibly to further new innovation. Note that, at the aggregate level, the average effective information propagation rate is $S \epsilon$ and consequently, having set the cardinality of the cognitive neighbourhood to $S$, the effective control parameter of the diffusion process is $\epsilon$. There are two forces at work in the information diffusion dynamics that we envisage: one is the lengthy accumulation of informational bits, a process that slows down and curbs diffusion, the other is the effective strength of propagation of the informational content of successful innovations which, on the contrary, enhances it. For $\epsilon<1$, some information accumulated at the single firm level is lost in the transmission process and consequently the propagation effect is too weak compared with the information accumulation 
effect: on average, only $S \epsilon$ firms receive a useful informational bit whereas $S$ had been cumulated by the innovating firm; information broadcasting is therefore dampened. For $\epsilon>1$ the information propagation effect is stronger than the one due to accumulation since it applies, on average, to $S \epsilon$ firms, i.e. to more firms than $S$. If $\epsilon=1$ there is no loss in the diffusion process, information being transmitted without hindrance to other firms. We later argue that $\epsilon \rightarrow 1$ is the interesting case, stressing the degree to which spillovers occur depending on structural characteristics, e.g. the cognitive density effect $J$, and on the searching effort put in place by firms.

We describe diffusion dynamics by using master equations. At the aggregate level, $\widehat{\phi}_{t}$ is the firms' transition rate from one state to the next, with $\widehat{\phi}_{t}=\widehat{q}_{t}+S \widehat{\epsilon}_{t} \rho_{S}$ in which the first term represents the arrival of exogenous information while the second term represents endogenous, localised spillovers. The system's time evolution is then given by the following differential equations (we omit time dependence for simplicity) ${ }^{6}$ :

$$
\begin{gathered}
\dot{\rho}_{S}=-\rho_{S}+\widehat{\phi}_{t} \rho_{S-1} \\
\dot{\rho}_{k}=-\widehat{\phi}_{t} \rho_{k}+\widehat{\phi}_{t} \rho_{k-1} \\
\dot{\rho}_{0}=-\widehat{\phi}_{t} \rho_{0}+\rho_{S}
\end{gathered}
$$

The equations RHS first term in (19) represents the outflow from state $k$, while the second represents the inflow, $k=0,1, \ldots, S$. Thus, the first equation's first term indicates the exit from

\footnotetext{
${ }^{6}$ We briefly sketch a heuristic derivation of the master equation for $\rho_{k}, k \in(1, S-1)$. The dynamics for $\rho_{S}$ and $\rho_{0}$ can be obtained in a similar way. Let $N_{k}=\rho_{k} J$ denote the average number of firms being in state $k$. $N_{k}$ changes either because of an outflux of firms, that is firms switching from state $k$ to $k+1$ or because of an influx of firms, that is firms switching from state $k-1$ to state $k$. This may happen either because firms acquire new and exogenous information, or because they capture an informational spill-over from congnitively neighbouring firms. Consider the outflux first. At the aggregate level, over a small time interval $\Delta t$, a firm discovers new information with an average probability $\widehat{q}_{t} \Delta t$, and hence, on average, $\widehat{q}_{t} N_{k} \Delta t$ firms switch to state $k+1$. Moreover, since over a small time period $\Delta t$, on average, $\widehat{\epsilon}_{t} S N_{s} \Delta t$ informational bits are broadcasted throughout the economy, $\widehat{\epsilon}_{t} S N_{s} \rho_{k} \Delta t$ firms in state $k$ are on average affected by this information diffusion process. A similar argument applies for firms switching from state $k-1$ to state $k$, which corresponds to the influx of firms into state $k$. Hence, the change in the number of firms being in state $k$ over a small time period $\Delta t$ is

$$
\Delta N_{k}=-\left(\widehat{q}_{t} N_{k}+\widehat{\epsilon}_{t} S N_{s} \rho_{k}\right) \Delta t+\left(\widehat{q}_{t} N_{k-1}+\widehat{\epsilon}_{t} S N_{s} \rho_{k-1}\right) \Delta t
$$

Note that $J$ is constant over time. Dividing both sides of this equation by $J \Delta t$ and letting $\Delta t \rightarrow 0$ we obtain the result.
} 
the cumulation process: all firms that have completed it introduce a new innovation and leave the scene to a new follower who starts the collection process from the very beginning. Consequently, an identical density appears as an influx in the last equation's last term.

The normalisation condition requires that:

$$
\sum_{k=0}^{S} \rho_{k}=1
$$

It is important to notice that this model features two time scales, namely the exogenous arrival of information on the one hand and its endogenous propagation on the other. We assume that the diffusion process takes place at a much faster pace than the discovery of new valuable exogenous information. In particular, we consider diffusion dynamics to be istantaneous compared with the time scale set by the exogenous arrival. This infinite time scale separation avoids the problem of the overlapping of innovation waves. Formally, this result is obtained in the limit of $\mu \rightarrow 0$, which corresponds to $\widehat{q} \rightarrow 0$.

Given that an informational bit has been discovered by a single firm at time $t$, let $V_{t}$ denote the number of firms succeeding in introducing an innovation. We call $V_{t}$ an innovation avalanche. $V_{t}$ is clearly a stochastic variable and its first two moments will be later characterised in the case of steady state equilibrium.

\subsection{Optimal search for innovation}

At the single industry level the probability of a would-be innovator of receiving, over a small time period $d t$, either exogenously or endogenously an informational bit is $\phi_{j, t} d t$, with $\phi_{j, t}=$ $\left(q_{j, t}+\epsilon_{j, t} S \rho_{S}\right)$ and where $q_{j, t}$ and $\epsilon_{j, t}$ are the sector specific values. Note that at the aggregate level, over the time period $d t, \epsilon_{j, t} S \rho_{S}$ is the number of firms receiving an innovation spill-over. By using Assumption 3, we are allowed to define the probability that firm $j$ receives an information shock over time period $d t$ as:

$$
\phi_{j, t}=q\left(h_{j, t}^{I_{1}}\right)+\epsilon\left(J, h_{j, t}^{I}-h_{j, t}^{I_{1}}\right) S \rho_{S}
$$


Note that $\rho_{S}$ in (20) does not depend on the firm's in-house R\&D investment. This is due to the fact that once the firm succeeds in introducing a new innovation, switching to state $S$, it is no longer involved in innovation searching but begins to produce and supply a new intermediate good leaving its place to be filled by a new follower that starts a new information collection process.

It is conceivable that firms engaged in an innovation search attempt to allocate a given amount of employment $h^{I}$ with the aim of increasing $\phi_{j, t}$ as much as possible. This assumed behaviour can be stylized by stating that they tend to maximize it by choosing a suitable $h_{j, t}^{I_{2}}$ for any given interaction strength $\epsilon_{j, t}$.

Lemma 1. On account of Assumption 3, (i) the size of specialised employment that is worth hiring to foster innovative advancement is $h_{j, t}^{I_{1}}=g^{J}\left(h_{j, t}^{I}\right)$ and $h_{j, t}^{I_{2}}=h_{j, t}^{I}-h_{j, t}^{I}$ with $0<g_{h^{I}}^{J}<$ 1, for each $J$; (ii) $\phi_{j, t}=\phi^{J}\left(h_{j, t}^{I}\right)$ exhibits the following properties: $\phi^{J}(0)=0, \phi_{h^{I}}^{J}\left(h^{I}\right)>0$, $\phi_{h^{I}, h^{I}}^{J}\left(h^{I}\right)<0$, and $\lim _{h^{I} \rightarrow 0} \phi_{h^{I}}^{J}\left(h^{I}\right)=\infty$, for each $J$.

\section{The rate of innovation}

The follower's outlook is to attempt to maximize the expected future profits that are likely to accrue as a consequence of an innovation which is, in turn, the result of the employment of a consistent amount of specialised labour $h_{j, t}^{I}$. In order to achieve this task, the would-be innovator must be able to forecast the expected period of time during which future profits will indeed be forthcoming, namely the period of time that he or she will remain a monopolist.

To proceed with determining expected profits and the rate of innovation, it is expedient to set forth two assumptions that correspond to what may be called commonly observed albeit stylised facts. The first, a well evidenced one, is that in modern industialised economies the wage rate follows productivity increases. For this reason, we make the following assumption:

Assumption 4: $\frac{w_{t_{2}}^{h}}{w_{t_{1}}^{h}}=\exp \left[\lambda m\left(t_{1}, t_{2}\right)\right]$, where $m\left(t_{1}, t_{2}\right) \equiv \frac{1}{J} \sum_{j=1}^{J}\left(n_{j, t_{2}}-n_{j, t_{1}}\right), t_{1}<t_{2}$, $m\left(t_{1}, t_{2}\right)$ is the average number of innovations per sector introduced in the entire economy after 
time $t_{1}$ and before time $t_{2}$.

The second deals with the follower's and would-be innovator's employment policy. It is quite reasonable to assume that once hired, the highly specialised manpower required to sustain the innovation-searching procedures be retained until the full completion of the whole time process. this statement serves the purpose of underlining the fact that each person is necessary and that there is no redundancy all along the process. We, accordingly, make a further assumption about the information accumulation process.

Assumption 5: Each firm keeps $h_{j, t}^{I}$ constant along the information accumulation process.

Note that, since at the aggregate level the workforce is constant, and since $h_{t}^{x}$ and $l_{t}$ are in fixed proportions, a consequence of Assumption 5 is that also $h_{t}^{x}$ remains constant.

On the strength of (18) and Assumptions 4 and 5, it is possible to calculate the expected profits of a successful innovation by taking into account future proceeds streaming from production and sale of the intermediate good in the period between two innovations as well as the cost of producing it. Normalizing, for simplicity's sake, the risk-free interest rate to zero the following result can be proved. ${ }^{7}$

Proposition 1 A potential innovator in sector $j$ at time $t$ expects to earn an amount of average proceeds over an expected time period $E_{t}\left[T\left(j, n_{j, t}+1\right)-t \mid T\left(j, n_{j, t}\right)=t\right]$ equal to $c \delta l_{t} w_{t}^{I} \Lambda_{j, t}$ where $\Lambda_{j, t}$ :

$$
\Lambda_{j, t}=E_{t}\left\{\int_{t}^{T\left(j, n_{j, t}+1\right)} \exp [\lambda m(t, \tau)] d \tau \mid T\left(j, n_{j, t}\right)=t\right\}
$$

On account of Assumption 5 and our symmetry assumptions this expression reads

$$
\Lambda_{j, t}=\frac{1}{\rho_{S-1} \phi^{J}\left(\overline{h_{j, t}^{I}}\right)-(J-1) \widehat{q} \rho_{S-1}\left[1-\frac{E_{t}\left(V_{t}\right)}{J}\right]\left\{E_{t}\left[\exp \left(\lambda \frac{V_{t}}{J}\right)\right]-1\right\}}
$$

where $\overline{h_{j, t}^{I}}$ indicates the expected number of workers employed in R\&D by the prospective new follower.

7 Had we used a micro-founded aggregate demand by using an utility function, thus considering consumers as facing an intertemporal coumption problem, we would have used the interest rate to equate consumption and production paths. 
$\Lambda_{j, t}$ measures the effects of expected productivity gain. It is interesting to note that it is decreasing in the probability $\phi^{J}\left(\overline{h_{j, t}^{I}}\right)$ Indeed, for $\frac{E_{t}\left(V_{t}\right)}{J} \rightarrow 0$, the case in which avalanches do not emerge and the effects of productivity gains are negligible, $\Lambda_{j, t}$ equals the mean waiting time for the follower to innovate. If $\frac{E_{t}\left(V_{t}\right)}{J}>0$ and avalanches are not negligible at the aggregate level, the denominator second addendum catches the effects of average aggregate productivity increases due to global interaction. In any case, $\Lambda_{j, t}$ is larger if avalanches emerge. It is also clear that $\Lambda_{j, t}$ is larger the larger is the variance of $V_{t}$, while the effect of an increase of $\frac{E_{t}\left(V_{t}\right)}{J}$ is not a priori obvious since, as $\frac{E_{t}\left(V_{t}\right)}{J}$ approaches $1, \Lambda_{j, t}$ approaches again the mean waiting time for the follower to innovate:

This is a significant result since it highlights the forces that are at play to generate the expectation of a productivity gain and, in turn, of average profits. A trade-off balances out the likelihood of an innovation coming to pass within the industry, thus ending the future monopolist's position, an event measured by $\rho_{S-1} \phi^{J}\left(\overline{h_{j, t}^{I}}\right)$, and the positive, profit increasing effects due to innovations occurring in industries other than the future monopolist's own. The latter effect is however bounded by some threshold. At this point the likelihood of being involved in the avalanche and of profits being consequently wiped out overcomes the effect due to the latter occurring only in sectors other than the future monopolist's own which would imply a profit rise due to an aggregate demand increase. It also serves the purpose to illustrate that effective technical progress results from contrasting forces and that likely productivity increases can partially be offset by the outlook of capital losses due to the loss of the leading position.

Realising profits implied by (21), the expectation of which depends on the likely period during which monopoly is enjoyed, is clearly subject to the probability $\rho_{S-1} \phi^{J}\left(\overline{h_{j, t}^{I}}\right)$ that in turn depends on the magnitude of the total manpower, in fact brainpower, $\overline{h_{j, t}^{I}}$, specifically employed for the purpose. The problem is then specified, given (21), by the following equation:

$$
\max _{h_{j, t}^{I}} w_{t}^{h}\left[\phi^{J}\left(h_{j, t}^{I}\right) h_{t}^{x} c \rho_{S-1} \Lambda_{j, t}-h_{j, t}^{I}\right]
$$


It is important to note that the solution of this maximum problem for the specialised labour force $h_{j, t}^{I}$ affords the would-be innovator to simultaneously influence the exogenous driving force through $h_{j, t}^{I_{2}}$ as well as the endogenous informational spillovers due to $h_{j, t}^{I_{1}}$. Moreover, note that in the maximization problem $(22)$ the firm takes $h_{t}^{x}$ as given since this is the specialised workers' employment to be hired once it succeeds in introducing the innovation. In the steady state, the latter corresponds to the amount of specialised workers employed by the leader.

\subsection{The steady state equilibrium}

The purpose of this paper is to investigate the properties of the model at the stationary (steady) state where $h_{i, t}^{I_{1}}=h_{j, t}^{I_{1}}=h^{I_{1}}$ and $h_{j, t}^{I_{2}}=h_{i, t}^{I_{2}}=h^{I_{2}}$ for each $i, j=1, \ldots, J$ and $t>0$, where $\widehat{\epsilon}=\epsilon=\epsilon\left(J, h^{I_{2}}\right), \widehat{q}=q=q\left(h^{I_{1}}\right)$ and $\overline{h^{I}}=h^{I}$. We accordingly proceed to determine the average avalanche size and its variance. We then solve for the equilibrium allocation of specialised manpower to be employed to generate an innovation.

Proposition 2 In the stationary state and in the limit for $\mu \rightarrow 0$, the first two moments of $V_{T}$ are:

$$
\begin{gathered}
E\left(V_{t}\right)=\left.\frac{\partial}{\partial q} \rho_{S}\right|_{q=0}=\frac{1}{(1-\epsilon) S} \\
E\left[\left(V_{t}\right)^{2}\right]=\left|\frac{\partial^{2}}{\partial q^{2}} \rho_{S}\right|_{q=0} \mid=2\left[\frac{1}{(1-\epsilon) S}\right]^{2}
\end{gathered}
$$

These results lend themselves to an intuitive interpretation of the system's properties. The system can in fact be viewed as receiving a single, small informational bit that eventually propagates throughout the economy setting off an innovation avalanche. The limit $\mu \rightarrow 0$ is a condition that amounts to assuming a very small, exogenous driving force establishing an infinite time scale separation between the discovery of a new bit of information and its endogenous propagation.

Since we are considering an economy where $J \rightarrow \infty$, the result stated in Proposition 2 is best interpreted by looking at the relative average avalanche dimension; a similar argument applies to its fluctuations, as shown in the next section. As long as $\epsilon<1$, the average avalanche size remains finite and it is consequently negligible compared with the system's dimension: aggregate innovation avalanches do not emerge. The intuition for this result is straightforward: the effective 
propagation mechanism is too weak compared with the information accumulation effect. For $\epsilon>1$ the system explodes since the effective propagation mechanism is too strong compared with the information accumulation mechanism. For $\epsilon \rightarrow 1$ such that $\frac{1}{(1-\epsilon) J} \rightarrow$ const $>0$, the system fluctuates around a critical state in which large innovation waves spanning the whole economy emerge.

For the following we consider

$$
\lim _{J \rightarrow \infty} \frac{1}{(1-\epsilon) J}=\theta
$$

For $\theta=0$, the case when innovation avalanches do not emerge, and for $\theta=\theta\left(h^{I_{2}}\right)=\theta\left(h^{I}-g^{J}\left(h^{I}\right)\right)>$ 0 , applying when they do, we posit $\theta_{h^{I_{2}}}\left(h^{I_{2}}\right)>0$; thus, simple algebra shows that $\theta_{h^{I}}=$ $\frac{\partial}{\partial h^{I}} \theta\left(h^{I}-g^{J}\left(h^{I}\right)\right)>0$. Note that the critical state $\theta>0$ is reached in the limit $\epsilon \rightarrow 1$, $\mu \rightarrow 0$ and $\frac{\mu}{1-\epsilon} \rightarrow 0$. Note further that, since $\frac{J \mu}{(1-\epsilon) J} \rightarrow \theta J \mu$, the condition $\frac{\mu}{1-\epsilon} \rightarrow 0$ implies that $J \mu \rightarrow 0 .{ }^{8} \quad \theta>0$ is the interesting case. It occurs when the economy in question possesses those properties insuring cohesion and the capability to interact, namely when the industrial district effect operates.

We are now in a position to characterise the asympotic values for the stationary state behavior of $\phi$.

Lemma 2. On account of Assumption 3, if avalanches do not emerge $(\theta=0), \lim _{J \rightarrow \infty} h^{I_{1}}=$ $\lim _{J \rightarrow \infty} g^{J}\left(h^{I}\right)=h^{I}$ and consequently $\phi=q\left(h^{I}\right)$, while if avalanches do emerge $(\theta>0)$, $\lim _{J \rightarrow \infty} h^{I_{1}}=\lim _{J \rightarrow \infty} g^{J}\left(h^{I}\right) \equiv g\left(h^{I}\right)<h^{I}$ with $\phi=\phi^{\infty}\left(h^{I}\right) \equiv \phi\left(h^{I}\right)$, where $\frac{\phi\left(h^{I}\right)}{J}=$ $q\left(g\left(h^{I}\right)\right) \theta\left(h^{I}\right)$

Example 1. A suitable and simple functional form for $\epsilon$ is $\epsilon\left(J, h^{I_{2}}\right)=\max \left\{d-\frac{\chi\left(h^{I_{2}}\right)}{J}, 0\right\}$, with $d \leq 1, \chi_{h^{I_{2}}}\left(h^{I_{2}}\right)<0$ and $\chi_{h^{I_{2}}, h^{I_{2}}}\left(h^{I_{2}}\right)>0 . \quad \chi\left(h^{I_{2}}\right)$ can be interpreted as the threshold for the number of firms insuring that information spillovers emerge. In this case the optimal

\footnotetext{
8 Note that we implicitly assume that the stationary state of the economy is never supercritical (i.e. with $\epsilon>1$ ) in which case the diffusion of information would lead to an autocatalytic process.
} 
equilibrium allocation of $h^{I_{1}}$ and $h^{I_{2}}$ is given by $\frac{q^{\prime}\left(h^{I_{1}}\right)}{q\left(h^{I_{1}}\right)}=-\frac{\chi_{h^{I_{2}}}\left(h^{I_{2}}\right)}{J(1-d)+\chi\left(h^{I_{2}}\right)}$ which yields $h^{I_{1}}=$ $g^{J}\left(h^{I}\right)$. Hence, if $d=1, \lim _{J \rightarrow \infty} h^{I_{1}}=g\left(h^{I}\right)<h^{I}$ the economy converges to a critical state where $\theta=\theta\left(h^{I_{2}}\right)=\frac{1}{\chi\left(h^{I}-g\left(h^{I}\right)\right)}$, while if $d<1, \lim _{J \rightarrow \infty} h^{I_{1}}=h^{I}$, i.e. $g\left(h^{I}\right)=h^{I}$, the economy converging to a subcritical state where $\theta=0$. Consequently, if avalanches do emerge, then $\lim _{J \rightarrow \infty} \frac{\phi^{J}\left(h^{I}\right)}{J}=\frac{q\left(g\left(h^{I}\right)\right)}{\chi\left(h^{I}-g\left(h^{I}\right)\right)}=\theta q$, while if they do not $\lim _{J \rightarrow \infty} \phi^{J}\left(h^{I}\right)=q\left(h^{I}\right)$.

Having characterised innovation avalanches, we turn to the equilibrium allocation of manpower. Whilst would-be innovators optimise their future profits by choosing to employ $h^{I}, \mathcal{L}=H^{I}+H^{x}+L$ must be satisfied in equilibrium. Equivalently, at the sector level $\ell=h^{I}+h^{x}+l$ with $\ell \equiv \frac{\mathcal{L}}{J}$. and thus, since $l=\frac{h^{x}}{\delta}, h^{x}=\frac{\delta}{1+\delta}\left(\ell-h^{I}\right)$

Proposition 3 There exists a unique equilibrium $h^{I}$ which increases in $\delta$ and $c$ but decreases in $S$.

This result has a clear economic interpretation. Being the ratio between the final and intermediate sector productivities and since final sector profits are zero by assumption, $\delta$, by increasing, raises the possibility for intermediate good producers to increase their price and thus their profits. As a result, innovations become more profitable and it pays to increase employment in research and development. It is straightforward that as the mark-up, $c$, increases innovations will also become more profitable with the same effect on innovation-sustaining employment. Finally, a higher $S$ lengthens the information collection process making searching more costly thus lowering the profitability of introducing innovations and the incentive of employing people to carry out the job.

Corollary 1. Compared with the case when at the aggregate level innovation avalanches do not emerge, $h^{I}$ is smaller when they do.

Hence, if innovation avalanches emerge, the positive feedback effect of aggregate demand is overcome by the negative one set by an increasing probability of avalanches involving the firm's 
sector; a fact that decreases $h^{I}$.

\section{The long-term growth-rate}

Taking the above generated results into account, more particularly, the expected size of an avalanche as determined by the equilibrium level of innovation-related employment, it is quite immediate to state the productivity-driven long-term expected growth rate of aggregate final output $Y_{t}$. On account of (14), the latter can be posited as follows:

$$
E_{t}\left[\ln \left(\frac{Y_{t+\Delta t}}{Y_{t}}\right)\right]=E_{t}\left[\ln \left(\frac{w_{t+\Delta t}}{w_{t}}\right)\right]=\lambda E_{t}[m(t, t+\Delta t)]
$$

Given Assumption 4 where the meaning of $m(t, t+\Delta t)$ is defined as the average number of innovations per sector introduced between time periods $t$ and $t+\Delta t$, the long-term growth rate is simply the rate of productivity growth times this number. In turn, the latter results in a straightforward way from the expected equilibrium number of avalanches times their expected size. The former is $\frac{q\left(h^{I}\right)}{S} \Delta t$ whilst the latter follows from (23):

$$
\lim _{J \rightarrow \infty} E_{t}\left[\ln \left(\frac{Y_{t+\Delta t}}{Y_{t}}\right)\right]=\lambda \frac{q\left(g\left(h^{I}\right)\right)}{S} \theta\left(h^{I}-g\left(h^{I}\right)\right) \Delta t
$$

It is quite immediate to see from (24) that the main impact on the long-term growth rate, given the productivity rate of increase $\lambda$, comes from the size of manpower employed to carry out the search for innovation. The latter affects it both by positively increasing the number of avalanches that are likely to come to pass in an economy and by enhancing their size. On the contrary, it is also immediate to check that this rate is negatively impacted upon by the length of the information process, represented by $S$, required to finally achieve an innovation. The avalanche size plays a crucial role. If $\theta$ equals zero, namely if the avalanche size is negligible in relation to the system's, the diffusion effect is nil and the expected growth rate that owes to it is not significantly different from zero. In this case, those innovations that may occur remain trapped in the industry which has first introduced them or, at best, in a small neighborhood, with scarcely any spillover 
elsewhere. $^{9}$ Thus, economy-wide growth driven by technological progress takes place only when $\theta$ is positive and sufficiently large, in which case innovations become correlated and spread across the system. It is also interesting to stress that $S$, the number of bits required to finally obtain an innovation, weakens the expected growth rate on account of two parallel effects. A longer information length lowers the probability of ever being able to innovate but it also lessens the incentive to invest in the manpower that is conducive to doing so. Finally, since the latter event positively depends on the mark-up magnitude, $c$, and on $\delta$, the ratio of direct to indirect labour productivity, these two parameters clearly have a positive impact on the said rate by increasing the incentive of innovation-directed investment.

\subsection{Discussion}

It is now possible to infer the implied time pattern of final goods prices. Using (8):

$$
\frac{p_{j, t_{2}}}{p_{j, t_{1}}}=\frac{w_{t_{2}}}{w_{t_{1}}} \frac{a_{j, t_{1}}}{a_{j, t_{2}}}
$$

and given Assumption 4:

$$
\frac{p_{j, t_{2}}}{p_{j, t_{1}}}=e^{\lambda\left(m\left(t_{2}, t_{1}\right)-\left(n_{j, t_{2}}-n_{j, t_{1}}\right)\right)}
$$

where $m$ is again the average number of innovations per sector introduced in the entire economy after time $t_{1}$ and before time $t_{2}$, introduced in assumption 4. (25) indicates that prices of the final goods whose intermediate sectors have been able to introduce a larger number of innovations than the economy's average fall proportionally to the productivity increase differential. By the same argument, the relative prices of more innovative industries fall in terms of the slower growing ones. Nevertheless, the consumer price index (CPI) displays only fairly small oscillations:

$$
C P I=\frac{\sum_{j=1}^{J} y_{j, 0} p_{j, t}}{\sum_{j=1}^{J} y_{j, 0} p_{j, 0}}=\frac{\sum_{j=1}^{J} p_{j, t}}{\sum_{j=1}^{J} p_{j, 0}}=\frac{w_{t}}{w_{0}} \frac{\sum_{j=1}^{J} \frac{1}{b_{j, t}}}{J}=\frac{1}{J} \sum_{j=1}^{J} e^{\lambda\left[m(0, t)-n_{j, t}\right]}
$$

\footnotetext{
${ }^{9}$ Notice that this result follows from the assumption that, over the whole economy, at most just one informational shock takes place in each time period.
} 
In the long run $n_{j, t} \simeq m(0, t)$, hence the CPI remains approximately constant. In other words, since in the long run all industries are affected on average by the same number of innovations and all sectors are expected to be involved in several avalanches, all prices exhibit, on average, the same long-run behaviour. Thus, while the labour costs fall owing to productivity increases generated by innovation waves, wages increase in the same proportion. It follows that the same basket of consumption goods remains approximately constant in value, workers being able to purchase a larger one as their real wages rise.

\section{Summing up and Conclusions}

This paper has carried out an investigation on the impact of innovation investment, basically in human resources, on the structure and long-term growth of an economic system. The model that has been discussed provides a view of the economy in which there is a large number of industries. The latter are seen as composed by a final good-producing sector populated by a multitude of firms, hence competitive, and an associated vertically integrated sector led by a monopolist that provides technologically cutting edge, frontier intermediate goods. Besides this ruling monopolist, however, a follower, not yet a manufacturer, is active by investing in human resources to support a search for the next generation of innovations. What is at stake is the intermediate-good sector leadership in following periods; the successful technological search deciding who is going to be the next monopolist. Indeed, today's incumbent stands the chance of losing the leading position so far enjoyed, of seeing his or her profits wiped out and possibly of starting anew a search process until an eventual new innovation is found out.

The formal model that has been used posits very simple, linear production functions and even simpler consumption demand patterns. While final good prices are such as to render profits zero, intermediate goods are priced through a mark up on the only cost that is actually incurred, namely, labour. Eschewing all complications in demand and supply relations allows us to afford greater detail in modelling the searching process leading to innovations. The main thrust of this 
paper lies, in fact, in an account of firms' local interaction weaving into global effects, through effective demand, that drive the economy on a long-term growth path. Firms are seen as employing specialised manpower for the purpose of carrying out an in-house search as well as in order to gather valuable information from other firms' spillovers. This is a gradual and local process. It is local since firms are bounded by their cognitive capabilities and can reach up to useful information only within a narrowly defined neighbourhood. It is gradual since information that is obtained builds up to a consolidated state that only upon becoming critical finally yields a full fledged innovative intermediate good. Interaction is thus made to depend on a given measure of strength that is determined by an endogenous variable representing the number of employees hired for the purpose and complemented by two exogenous variables, namely the number of industries that compose the economy and a structural element that may eventually play the role of control parameter. The former insures contiguity of information- broadcasting across neighbourhoods, the latter accounts for positive externalities generated by cluster and industrial district effects. This treatment of the in-house, independent search, the idiosyncratic driving force, and information collection through interaction leads to a definition of the firms' probability to improve their state of knowledge towards achieving an innovation through the aggregate impact that all have on the process. A set of master equations depicting the gradual transition from a given state to a richer one allows to investigate the dynamic properties of interaction and define an asymptotically stable stationary state highlighting the role of the independent driving force on the share of firms capable of innovating. The properties of the diffusion process are accordingly derived as well as the average number of firms succeeding in introducing a new innovation given that a single exogenous informational bit has been contrived. Its variance is then calculated.

This paper stresses that the structural 'industrial district effect' is of importance and that the compelling task is then to determine the magnitude of a would-be innovator's investment as he or she attempts to challenge the ruling monopolist who extracts profits from a past innovation but does no searching. The framework in which this important result is obtained is fashioned as 
an attempt to maximise the probability of achieving an innovation by a suitable size of dedicated employment. The latter is made to depend on the expected flow of profits that are likely to accrue in the case of a successful innovation given the average innovation wave waiting time. It is exactly at this stage that the full impact of global, rather than local, interaction is brought in full light. A would-be innovator has to take into account the contrasting effects that are the outcome of success. Innovations increase productivity and clearly this affects future profits. Quite importantly, the expected average aggregate impact of innovations in sectors other than in the would-be innovator's own will increase the future stream of profits through demand effects.

A long-term growth rate straightforwardly results as a positive function of employment devoted to innovation generation. The derivation of the expected long-term growth rate incorporates the main results that have been derived. Crucially, it depends on the emergence of innovation waves. For this event to come to pass the economy must necessarily be densely populated by industries. This is because only if knowledge overlapping takes place spillovers can effectively spread across the system. To this effect, clustering and 'industrial districts' are relevant since they insure that industries lie within information-wise dense networks. They both concur to determining the strength with which information is broadcast. If too weak, innovations, if they occur, remain haphazard events of no consequence for the system as a whole; if critical, avalanches emerge and become economy-wide phenomena.

Productivity growth naturally impacts upon prices. They are shown to be lower where innovations have occurred in relation to where they have not and this causes profits to rise in consequence. This is all the more so, the greater is the average size of the avalanche. Yet, innovations that will take place where he or she will stand as a monopolist will suffice to oust him or her out of the market and exhaust profits. There is, indeed, a trade off between local and global effects. The paper accordingly stresses this important point as a product of the interplay of two contrasting forces and it shows that flows of expected profits are dependent on the size of innovation-oriented employment. Finally, it is shown that as prices vary in proportion to productivity, the consumer 
price index remains constant, a larger purchasing power being afforded by higher real wages.

\section{Appendix}

Proof of Lemma 1. The first order condition for this problem is

$$
q^{\prime}\left(h_{j, t}^{I_{1}}\right)=S \rho_{S} \frac{\partial}{\partial h_{j, t}^{I_{2}}} \epsilon\left(J, h_{j, t}^{I}-h_{j, t}^{I_{1}}\right)
$$

Given (20), and the envelope theorem, $\phi_{h^{I}}^{J}\left(h^{I}\right)=\epsilon_{h^{I_{2}}}\left(J, h^{I_{2}}\right) S \rho_{S}$. It follows from Assumption 3 that $\phi^{J}(0)=0, \phi_{h^{I}}^{J}\left(h^{I}\right)>0, \lim _{h^{I} \rightarrow 0} \phi_{h^{I}}^{J}\left(h^{I}\right)=\infty$ and $\lim _{h^{I_{2} \rightarrow 0}} \epsilon_{h^{I_{2}}}\left(J, h^{I_{2}}\right) S \rho_{S}=\infty$ and $\phi_{h^{I}, h^{I}}^{J}\left(h^{I}\right)<0$.

Proof of Proposition 1. Consider $\Lambda_{j, t}$ as the expected present value of future productivity increases for the follower of a sector, say $j$. In order to calculate the expected profit of an innovation for a given sector follower, say $j$, given that he or she succeeds in introducing an innovation at time $t$,

$$
\Lambda_{j, t}=E_{t}\left\{\int_{t}^{T\left(j, n_{j, t}+1\right)} \exp [-r(\tau-t)] \exp [\lambda m(t, \tau)] d \tau \mid T\left(j, n_{j, t}\right)=t\right\}
$$

where $r$ is the risk-free interest rate; we split the integral into two terms

$$
\begin{aligned}
\Lambda_{j, t}= & E_{t}\left\{\int_{t}^{\Delta t+t} \exp [-r(\tau-t)] \exp [\lambda m(t, \tau)] d \tau \mid T\left(j, n_{j, t}\right)=t\right\}+ \\
& E_{t}\left\{\int_{\Delta t+t}^{T\left(j, n_{j, t}+1\right)} \exp [-r(\tau-t)] \exp [\lambda m(t, \tau)] d \tau \mid T\left(j, n_{j, t}\right)=t\right\} .
\end{aligned}
$$

Since $m(t, \tau)=m(t, t+\Delta t)+m(t+\Delta t, \tau)$

$$
\begin{gathered}
\Lambda_{j, t}=\int_{t}^{\Delta t+t} \exp [-r(\tau-t)] E_{t}\left\{\exp [\lambda m(t, \tau)] \mid T\left(j, n_{j, t}\right)=t\right\} d \tau+ \\
\exp (-r \Delta t) E_{t}\left\{\exp [\lambda m(t, t+\Delta t)] \int_{\Delta t+t}^{T\left(j, n_{j, t}+1\right)} \exp \{-r[\tau-(t+\Delta t)]\} .\right. \\
\left.\exp [\lambda m(t+\Delta t, \tau)] d \tau \mid T\left(j, n_{j, t}\right)=t\right\}
\end{gathered}
$$

Now we estimate both terms in the r. h. s. in the limit when $\Delta t \rightarrow 0$. The probability that an innovation wave arrives somewhere in the system in the short time span between $t$ and $t+\Delta t$ without involving sector $j$ is $(J-1) \widehat{q} \rho_{S-1}\left[1-\frac{E_{t}\left(V_{t}\right)}{J}\right] \Delta t$ and consequently $E_{t}\left\{\exp [\lambda m(t, \tau)] \mid T\left(j, n_{j, t}\right)=t\right\}=$ 
$\left\{1-(J-1) \widehat{q} \rho_{S-1}\left[1-\frac{E_{t}\left(V_{t}\right)}{J}\right](\tau-t)\right\}+(J-1) \widehat{q} \rho_{S-1}\left[1-\frac{E_{t}\left(V_{t}\right)}{J}\right](\tau-t) E_{t}\left[\exp \left(\frac{\lambda}{J} V_{t}\right)\right]$, whence $\int_{t}^{\Delta t+t} \exp [-r(\tau-t)] E_{t}\left\{\exp [\lambda m(t, \tau)] \mid T\left(j, n_{j, t}\right)=t\right\} d \tau=\Delta t[1+o(1)]$ as $\Delta t \rightarrow 0$. Turning to the second term, we have

$$
\begin{gathered}
E_{t}\left\{\exp [\lambda m(t, t+\Delta t)] \mid T\left(j, n_{j, t}\right)=t\right\} \\
=1-(J-1) \widehat{q} \rho_{S-1}\left[1-\frac{E_{t}\left(V_{t}\right)}{J}\right] \Delta t+(J-1) \widehat{q} \rho_{S-1}\left[1-\frac{E_{t}\left(V_{t}\right)}{J}\right] \Delta t E_{t}\left[\exp \left(\frac{\lambda}{J} V_{t}\right)\right] \\
E_{t}\left\{\int_{\Delta t+t}^{T\left(j, n_{j, t}+1\right)} \exp \{-r[\tau-(t+\Delta t)]\} \exp [\lambda m(t+\Delta t, \tau)] d \tau \mid T\left(j, n_{j, t}\right)=t\right\}=E_{t}\left(\Lambda_{j, t+\Delta t}\right)
\end{gathered}
$$

and, neglecting correlations,

$$
\begin{gathered}
\Lambda_{j, t}=\left\{( 1 - r \Delta t ) \left\{1-(J-1) \widehat{q} \rho_{S-1}\left[1-\frac{E_{t}\left(V_{t}\right)}{J}\right]+\right.\right. \\
\left.\left.(J-1) \widehat{q} \rho_{S-1}\left[1-\frac{E_{t}\left(V_{t}\right)}{J}\right] \Delta t E_{t}\left[\exp \left(\frac{\lambda}{J} V_{t}\right)\right]\right\}\right\} E_{t}\left[\Lambda_{j, t+\Delta t} \mid T\left(j, n_{j, t}\right)=t\right]+o(\Delta t)+\Delta t \\
=\left\{1-(J-1) \widehat{q} \rho_{S-1}\left[1-\frac{E_{t}\left(V_{t}\right)}{J}\right]+(J-1) \widehat{q} \rho_{S-1}\left[1-\frac{E_{t}\left(V_{t}\right)}{J}\right] \Delta t E_{t}\left[\exp \left(\frac{\lambda}{J} V_{t}\right)\right]-r \Delta t\right\} . \\
E_{t}\left[\Lambda_{j, t+\Delta t} \mid T\left(j, n_{j, t}\right)=t\right]+o(\Delta t)+\Delta t
\end{gathered}
$$

On the other hand, the capital loss in the case of an innovation from the sector's follower is the entire flow of profit $\Lambda_{j, t}$, which occurs with the probability, $\phi^{J}\left(\overline{h_{j, t}^{I}}\right), \overline{h_{j, t}^{I}}$ being the expected number of workers employed in R\&D by the new follower, that the average would-be innovator be in a neighbourhood in which a firm is involved in an innovation-relevant event. Using this,

$$
E_{t}\left[\Lambda_{j, t+\Delta t} \mid T\left(j, n_{j, t}\right)=t\right]-\Lambda_{j, t}=-\phi^{J}\left(\overline{h_{j, t}^{I}}\right) \rho_{S-1} \Delta t \Lambda_{j, t}
$$

and substituting and passing to the limit as $\Delta t$ goes to 0 , we obtain

$$
\Lambda_{j, t}=\frac{1}{r+\phi^{J}\left(\overline{h_{j, t}^{I}}\right) \rho_{S-1}+(J-1) \widehat{q} \rho_{S-1}\left[1-\frac{E_{t}\left(V_{t}\right)}{J}\right]\left\{1-E_{t}\left[\exp \left(\frac{\lambda}{J} V_{t}\right)\right]\right\}}
$$

Normalizing the risk-free interest rate to zero we obtain the expression in the text.

Proof of Proposition 2. Equilibrium values can be maintained over time if and only if the stock of information existing in the system remains constant. On the one hand, at every $d t$ the exogenous influx of information, generated by followers' in-house research is $q\left(1-\rho_{S}\right) J d t$, while information interaction generates an endogenous diffusion of $J \varepsilon S \rho_{S} d t$. On the other hand, 
at every instant $J \rho_{S} d t$ active firms manage to innovate and, in so doing, oust the leaders of their respective industries, thus destroying their whole stock of information $J S \rho_{S} d t$. Positing equality between the information generated by followers' search and that destroyed by innovations, we obtain the equilibrium condition in the form

$$
q\left(1-\rho_{S}\right)+\varepsilon S \rho_{S}=S \rho_{S}
$$

Whence ${ }^{10}$

$$
\rho_{S}=\frac{q}{(1-\epsilon) S+q}
$$

Applying the formulas for the first and the second moment of the avalanche distribution given by Vespignani and Zapperi (1998) and Andergassen (2001), respectively, the results stated in the proposition follow ${ }^{11}$.

Proof of Lemma 2. Consider the first order condition for the maximisation problem of $\phi$ (26). Neglecting higher order terms we have $\rho_{S}=\frac{q}{s(1-\epsilon)}$ and hence $((26)$ reads

$$
q^{\prime}\left(h^{I_{1}}\right)=q\left(h^{I_{1}}\right) \frac{\epsilon_{h^{I_{2}}}\left(J, h^{I}-h^{I_{1}}\right)}{1-\epsilon}=\theta q\left(h^{I_{1}}\right) J \epsilon_{h^{I_{2}}}\left(J, h^{I}-h^{I_{1}}\right)
$$

Using Assumption 3, in the limit of $J \rightarrow \infty$ we obtain that $\frac{q^{\prime}\left(h^{I_{1}}\right)}{q\left(h^{I_{1}}\right)} \sim \theta$, and hence if $\theta=0$, $h^{I_{1}}=h^{I}$, while if $\theta>0, h^{I_{1}}=g\left(h^{I}\right)<h^{I}$.

To complete the proof we calculate $\phi$. If avalanches do not emerge, $\phi$ is trivially equal to $q$. If avalanches do emerge, then, since $\rho_{S-1}=\frac{1}{S}, \phi=q+\frac{\epsilon}{1-\widehat{\epsilon}} \widehat{q} \cdot{ }^{12} \quad$ Moreover, since $\widehat{q}=q$ and $\widehat{\epsilon}=\epsilon$, we obtain $\phi=\frac{q}{1-\epsilon}$. Substituting into this expression $\theta$ the result is obtained.

Proof of Proposition 3. Note that, because of Assumption 5 and the fact that expected values are constant over time, $\Lambda_{j, t}$ also remains constant and, moreover, because of our symmetry assumption, all sectors face the same problem.

\footnotetext{
10 Solving the model (19) for the stationary state and, consistently with the mean-field approach that we have followed, ignoring higher order terms, the same result can be obtained.

11 It is easy to see that the steady state is asymptotically stable (see Vespignani and Zapperi, 1998, for details).

12 As mentioned above, we neglect terms higher than the first order in $q$.
} 
Consider first the case where avalanches do emerge. From the first order condition of (22) and for $J \rightarrow \infty$ it follows that

$$
\phi_{h^{I}}\left(h^{I}\right) h^{x}=\frac{S}{c \Lambda}
$$

where, since $\lim _{J \rightarrow \infty} \frac{\phi^{J}\left(h^{I}\right)}{J}=\theta q=\theta\left(h^{I}-g\left(h^{I}\right)\right) q\left(g\left(h^{I}\right)\right)>0$,

$$
\Lambda=\frac{1}{\phi\left(h^{I}\right) \frac{1}{S}\left[1-\frac{\lambda}{S}\left(1-\frac{1}{S} \theta\right)\left(1+\frac{\lambda}{S} \theta\right)\right]}
$$

Rewriting the FOC (27) we obtain

$$
\Phi\left(h^{I}\right) \equiv \phi_{h^{I}}\left(h^{I}\right) c \frac{\delta}{1+\delta}\left(\ell-h^{I}\right)=\phi\left(h^{I}\right)\left[1-\frac{\lambda}{S}\left(1-\frac{1}{S} \theta\right)\left(1+\frac{\lambda}{S} \theta\right)\right] \equiv \Psi\left(h^{I}\right)
$$

where $\Phi^{\prime}\left(h^{I}\right)=\phi_{h^{I}, h^{I}}\left(h^{I}\right) c \frac{\delta}{1+\delta}\left(\ell-h^{I}\right)-\phi_{h^{I}}\left(h^{I}\right) c \frac{\delta}{1+\delta} h^{I}<0$, with $\lim _{h^{I} \rightarrow 0} \Phi\left(h^{I}\right)=\infty$ and $\Phi(\ell)=0$, and $\Psi^{\prime}\left(h^{I}\right)=\phi_{h^{I}}\left(h^{I}\right)\left[1-\frac{\lambda}{S}\left(1+\frac{\lambda}{S} \theta\right)\left(1-\frac{1}{S} \theta\right)\right]+\phi\left(h^{I}\right) \frac{\lambda}{S^{2}} \theta_{h^{I}} \cdot\left(1+\frac{\lambda}{S} \theta\right)-\phi\left(h^{I}\right)\left(1-\frac{1}{S} \theta\right)\left(\frac{\lambda}{S}\right)^{2} \theta_{h^{I}}$ which, for $\frac{\lambda}{S}$ sufficiently small, is positive, and with $\Psi(0)=0$ and $\Psi(\ell)>0$. Hence a unique $h^{I}$ solving $\Phi\left(h^{I}\right)=\Psi\left(h^{I}\right)$ exists. Since $\Phi\left(h^{I}\right)$ is increasing in $\delta$ and $c$, and $\Psi\left(h^{I}\right)$ is increasing in $S$, applying the implicit function theorem the comparative results can be established.

If avalanches do not emerge the first order condition reads

$$
q_{h^{I}}\left(h^{I}\right) h^{x}=\frac{1}{c} q\left(h^{I}\right)
$$

where, since $h^{x}=\ell-h^{I}$, the LHS of (28) is decreasing in $h^{I}$ with $\lim _{h^{I} \rightarrow 0} q_{h^{I}}\left(h^{I}\right) h^{x}=\infty$ and $\lim _{h^{I} \rightarrow \ell} q_{h^{I}}\left(h^{I}\right) h^{x}=0$ while the RHS of $(28)$ is increasing in $h^{I}$ with $q(0)=0$ and $q(\ell)>0$. Hence, a unique equilibrium exists.

Proof of Corollary 1. Note that if $\theta\left(h^{I}\right)=0$ as $J \rightarrow \infty$, that is if innovation avalanches do not emerge, then $\Psi\left(h^{I}\right)$ is larger and consequently $h^{I}$ is lower.

\section{References}

Aghion, P., Howitt, P., 1998. Endogenous Growth Theory. Cambridge, Mass.: Massachusetts Institute of Technology.

Aghion, P., Howitt, P., 1992. A model of growth through creative destruction. Econometrica $60,323-51$. 
Albert,R.,Barabasi, A.L.,2002. Statistical Mechanics of Complex Networks. Review of Modern Physics, 74, 47-97

Andergassen, R., Nardini, F., Ricottilli, M., 2005. Firms' interaction and technological paradigms. In J. Leskow, M. Puchet and L. F. Punzo (Eds.). New Tools for Economic Dynamics. Berlin: Springer Verlag Lecture Notes in Economics and Mathematical Systems.

Andergassen, R., Nardini, F., Ricottilli, M., 2006. Innovation Waves, Self-organized Criticality and Technological Convergence, Journal of Economic Behavior and Organization, 61, $710-728$.

Arenas, A., Díaz-Guilera, A., Pérez, C. J., Vega-Redondo, F., 2002. Self-organized criticality in evolutionary systems with local interaction. Journal of Economic Dynamics and Control $26,2115-2142$.

Arthur, W. B., 1994. Increasing returns and path dependence in the economy. (Ann Arbor: University of Michigan Press).

Bak, P., 1996. How Nature Works: The Science of Self-Organized Criticality. New York: Springer.

Bak, P., Chen, K., Scheinkman, J., Woodford, M., 1993. Aggregate fluctuations from independent sectoral shocks: self organized criticality in a model of production and inventory dynamics. Ricerche Economiche 47, 3-30.

Bak, P., Tang, C., Wiesenfeld, K., 1988. Self-organized criticality. Physical Review A 38, 364-374.

David, P. (1990), "The Dynamo and the Computer: An Historical Perspective on the Modern Productivity Paradox," American Economic Review 80, 355 - 361.

Dosi, G., 1988. Sources, procedures and macroeconomic effects of innovation. Journal of Economic Literature, 26, 1120-1171.

Fai, F., von Tunzelmann, N., 2001. Industry-specific competencies and converging technological systems: evidence from patents. Structural Change and Economic Dynamics 12, 141-170.

Helpman, E., Trajtenberg, M., 1998. A Time to Sow and a Time to Reap: Growth Based on General Purpose Technologies. Cambridge, Mass., MIT Press.

Jackson, M.O. (2008). Social Networks in Economics,forthcoming in the Handbook of Social Economics, edited by Benhabib J., A. Bisin, M. Jackson, Elsevier.

Jaffe, A., Trajtenberg, M. 2002. Patents, Citations and Innovations: A Window on the Knowlege Economy. Cambridge, Mass., MIT Press.

Mokyr, J. (1992). The Lever of Riches. Technological Creativity and Economic Progress. Oxford University Press.

Romer, P. M., 1990. Endogenous technical change. Journal of Political Economy 98,5.

Rosenberg, N., 1976. Perspectives on Technology. (Cambridge: Cambridge University Press).

Vespignani, A., Zapperi, S., 1998. How Self-organized criticality works: A unified mean-field picture. Physical Review E 57, 6345-6362.

Watts,D.J.,Strogatz,S.H., 1998. Collective Dynamics of Small World Networks. Nature. Vol.393, 440-442. 